$<$ Supporting Information>

\title{
Control of Interchain Contacts, Solid State Fluorescence Quantum Yield and Charge Transport of Cationic Conjugated Polyelectrolytes by Choice of Anion
}

\author{
Renqiang Yang, Andres Garcia, Dmitry Korystov, Alexander Mikhailovsky, \\ Guillermo C. Bazan* and Thuc-Quyen Nguyen*
}

Mitsubishi Chemical Center for Advanced Materials, Department of Chemistry \& Biochemistry, Institute for Polymers and Organic Solids, University of California, Santa Barbara, California 93106

Corresponding Authors: bazan@,chem.ucsb.edu, quyen@,chem.ucsb.edu

\section{X-ray Photoelectron Spectroscopy (XPS) Analysis}

XPS spectra were recorded on Kratos Axis Ultra XPS system with a base pressure of $1 \times 10^{-10}$ mbar (UHV), using monochromated Al K $\alpha$ X-ray source at $h v=1486 \mathrm{eV}$. The obtained binding energy (BE) of C1s was referenced to $284.5 \mathrm{eV}$. Figures S1-S4 show the XPS spectra of polyelectrolyte with $\mathrm{BF}_{4}^{-}, \mathrm{CF}_{3} \mathrm{SO}_{3}^{-}, \mathrm{PF}_{6}^{-}$and $\mathrm{BPh}_{4}^{-}$anions. The two peaks located at binding energy about 99 and $149 \mathrm{eV}$ assigned to Si2p and Si2s of the carbon adhesive. When the sample film was thinner, the two peaks of Si element were higher. In the XPS spectra of polyelectrolyte exchanged $\mathrm{Br}$ with different anions, the Br peaks nearly disappeared, and the corresponding exchange ions' elemental characteristic peaks were recorded. From atomic concentration ratios calculated from peak intensities using CasaXPS Version 2.3.5 software (using Scofield's cross-section values) ${ }^{1}$, more than $95 \%$ of bromide was exchanged. 


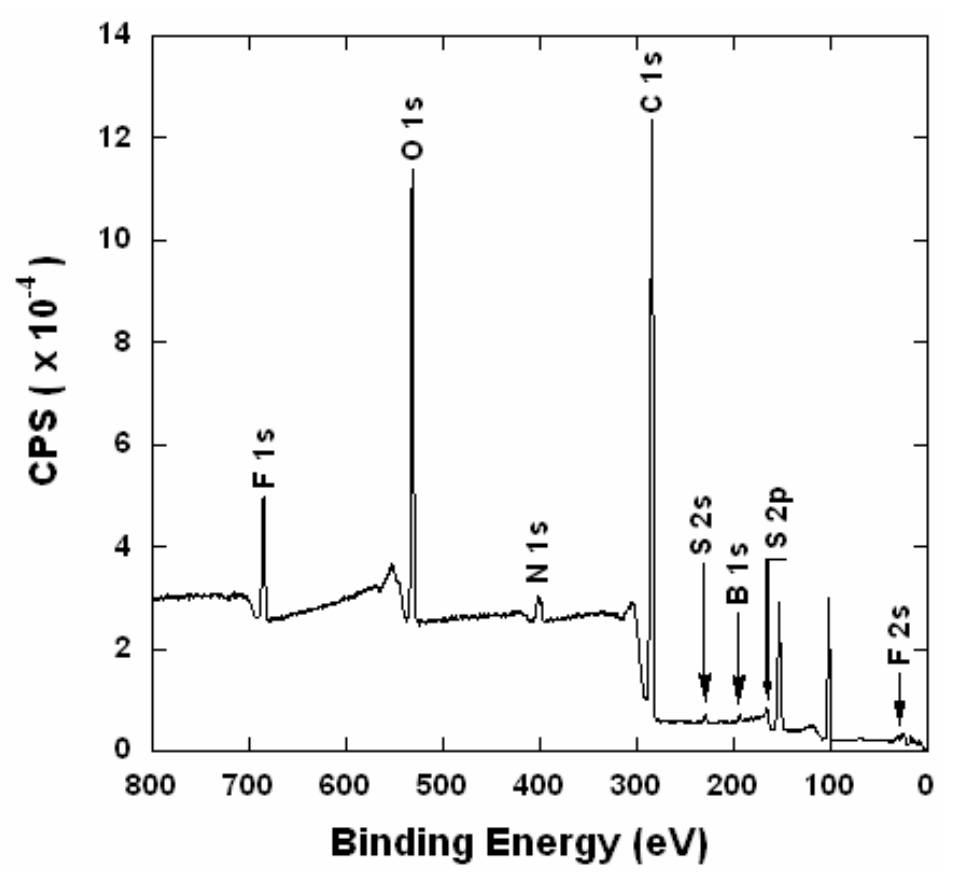

Figure S1. XPS spectra of polyelectrolyte PFBT-BF $_{4}$.

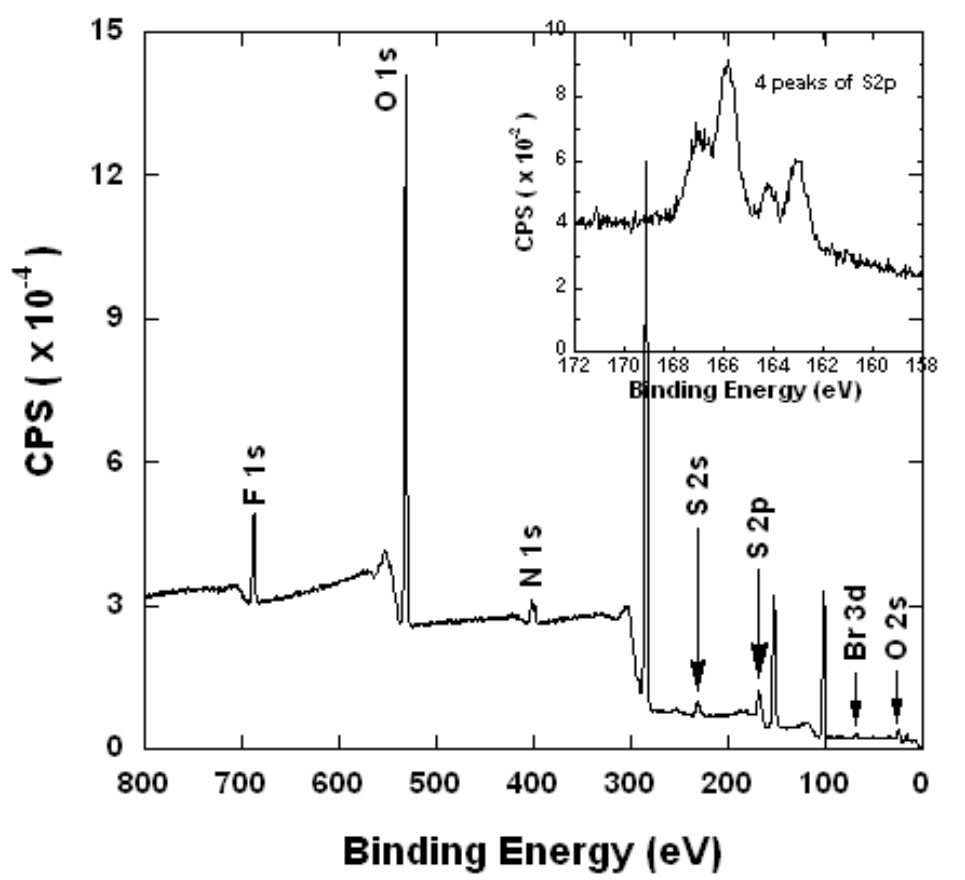

Figure S2. XPS spectra of polyelectrolyte PFBT-CF $\mathbf{S O}_{3}$ (insert: S2p amplified). 


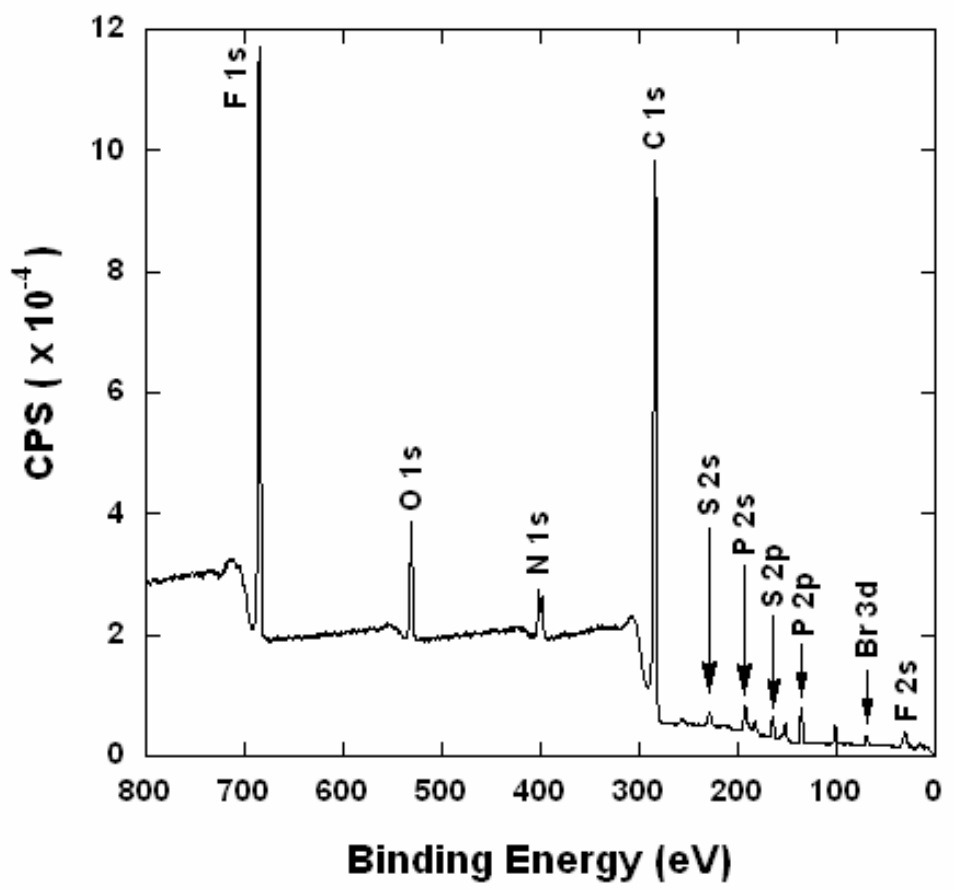

Figure S3. XPS spectra of polyelectrolyte PFBT-PF 6 .

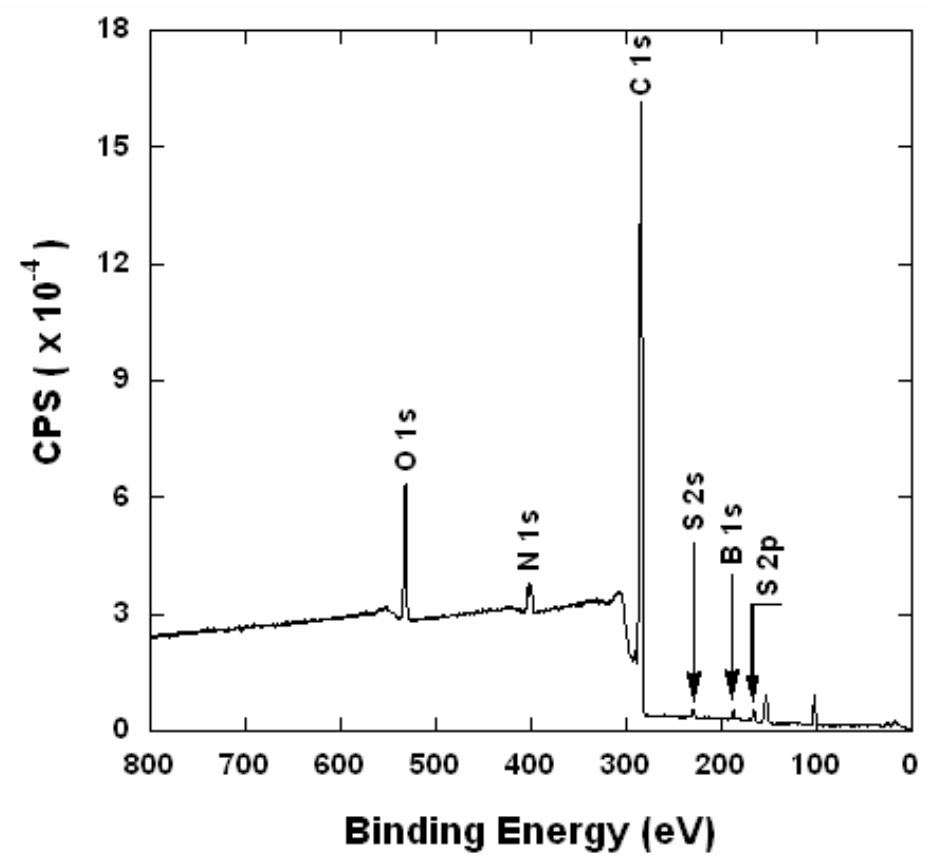

Figure S4. XPS spectra of polyelectrolyte PFBT-BPh . $_{4}$ 


\section{Dynamic Light-Scattering}

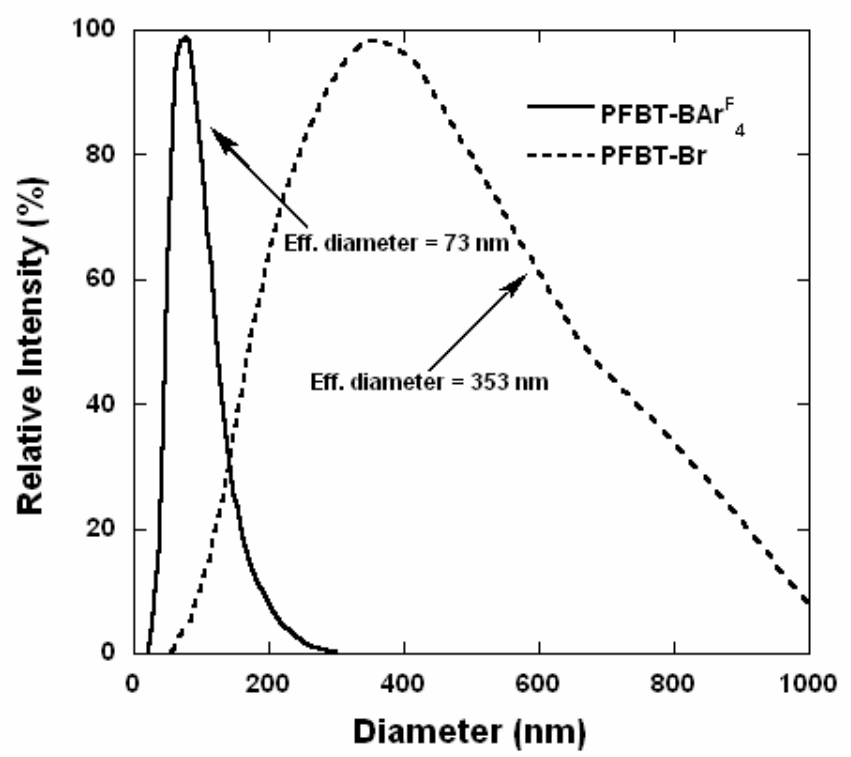

Figure S5. Size distributions (effective diameter) from dynamic light scattering for PFBT-Br (dashed curve) and PFBT-BAr ${ }_{4}{ }_{4}$ (solid curve) in deionized water.

\section{Conducting AFM}

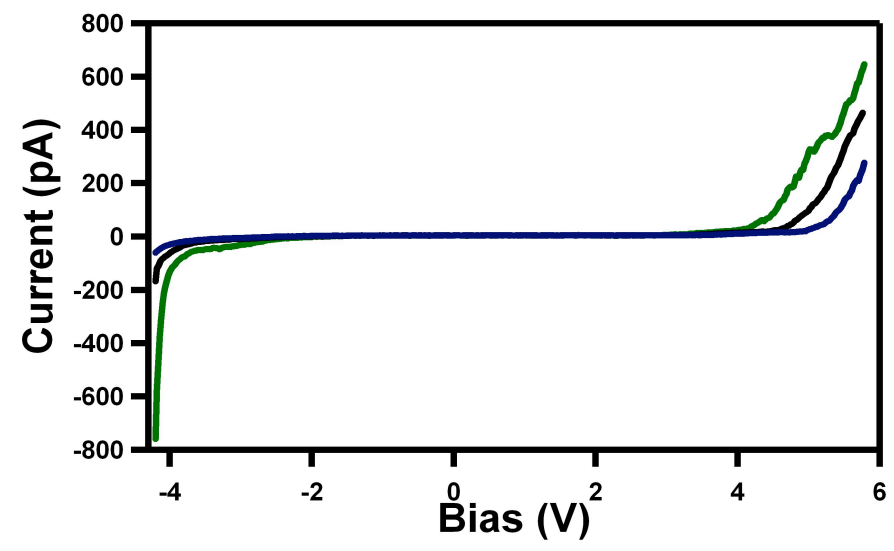

Figure S6. The average current-voltage curves of the PFBT-Br film. Forty I-V curves were collected from multiple locations of the film. The I-V curves fall into three current levels and the average I-V curve for each group is plotted: 18\% (green), 30\% (black), and 52\% (blue).

\section{Reference}

${ }_{1}^{1}$ Scofield, J. H. J. Electron Spectrosc. Relat. Phenom. 1976, 8, 129. 